\title{
The Insulator to Superconductor Transition in Ga-Doped Semiconductor Ge Single Crystal Induced by the Annealing Temperature
}

\author{
Y. B. Sun, ${ }^{1,2}$ Z. F. Di, ${ }^{1}$ T. Hu, ${ }^{1}$ and X. M. Xie ${ }^{1}$ \\ ${ }^{1}$ State Key Laboratory of Functional Materials for Informatics, Shanghai Institute of Microsystem and Information Technology, \\ Chinese Academy of Sciences, Shanghai 200050, China \\ ${ }^{2}$ University of Chinese Academy of Sciences, Beijing 100049, China
}

Correspondence should be addressed to T. Hu; thu@mail.sim.ac.cn

Received 26 December 2014; Revised 8 March 2015; Accepted 19 March 2015

Academic Editor: Viorel Sandu

Copyright (C) 2015 Y. B. Sun et al. This is an open access article distributed under the Creative Commons Attribution License, which permits unrestricted use, distribution, and reproduction in any medium, provided the original work is properly cited.

We have fabricated the heavily Ga-doped layer in Ge single crystal by the implantation and rapid thermal annealing method. The samples show a crossover from the insulating to the superconducting behavior as the annealing temperature increases. Transport measurements suggest that the superconductivity is from the heavily Ga-doped layer in Ge.

\section{Introduction}

Since the superconductivity was observed in metal in 1911, intensive efforts have been performed to find new superconductor such as high $T_{c}$ cuprate [1] and pnictide [2]. The application of superconductor on the traditional industry now motivates scientists to find the superconductivity in semiconductor materials, since it can solve the problem of power consumption of integrated circuit and improve the efficiency of the device. As the superconductivity is induced in the diamond doped with boron [3] and the silicon doped with boron [4], the Ga-implanted germanium [5] with a total dose of $2 \times 10^{16} \mathrm{~cm}^{-2} \mathrm{Ga}$ was recently found to show superconductivity at the low temperature with the superconducting transition temperature lower than the germanium heavily doped with $4 \times 10^{16} \mathrm{~cm}^{-2} \mathrm{Ga}$ [6]. The origin of superconductivity in the heavily doped semiconductors, however, is an open question yet, even after the intensive study [6-8]. In this work, we fabricated the heavily Ga-doped Ge with a $30 \mathrm{~nm} \mathrm{SiO}{ }_{2}$ cover layer by implanting $\mathrm{Ga}$ into the Ge single crystals and performed the rapid thermal annealing (RTA) on these samples. After performing the scanning electron microscope (SEM) and transport measurement, we find that the annealing temperature can induce an insulator to superconductor transition in Ga-doped Ge samples. Our study of superconductivity in the Ga-implanted Ge combines the superconductor and semiconductor which will contribute to application of superconductor on the traditional semiconductor industry.

\section{Experimental Details}

We chose the $(1,0,0)$ oriented and $\mathrm{P}$ doped germanium as the substrate in this experiment. On the top of the germanium we grew a $30 \mathrm{~nm} \mathrm{SiO}{ }_{2}$ cover layer through plasmaenhanced chemical vapor deposition (PECVD). After that, we implanted $4 \times 10^{16} \mathrm{Ga} \mathrm{cm}^{-2}$ at an ion energy of $100 \mathrm{keV}$ into it. Here, the $\mathrm{SiO}_{2}$ capping layer can prevent the surface degradation [6] except the implanted region during implantation as shown in Figure 1.

We annealed the Ga-doped Ge samples by the rapid thermal annealing (RTA) for $60 \mathrm{~s}$ in flowing Ar atmosphere at various temperatures. The RTA was reported to play an important role in recrystallizing the amorphous implanted layer, which leads to the formation of the highly Ga-doped layer at the $\mathrm{SiO}_{2} / \mathrm{Ge}$ interface $[6,8]$. We therefore investigate the surface structure of the samples by the scanning electron microscope (SEM). The surface degradation does not occur 


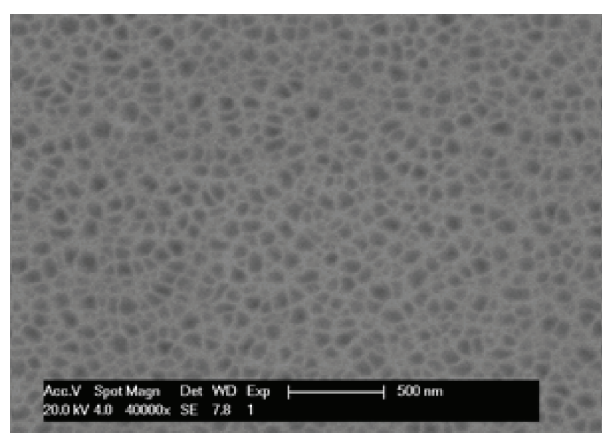

(a)

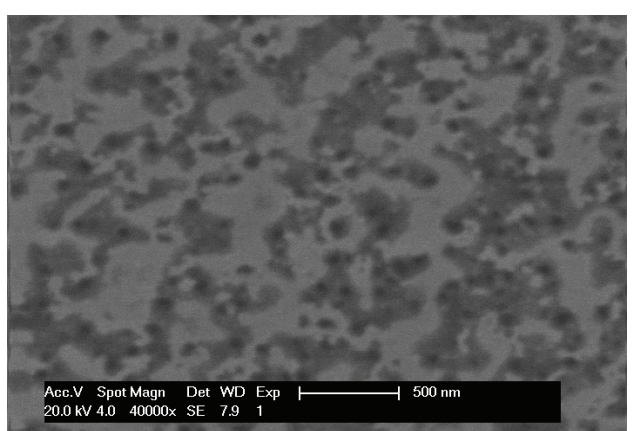

(b)

FIGURE 1: Surface structure investigated with SEM in the (100) oriented Ge single crystal, which exposed at $4 \times 10^{16} \mathrm{~cm}^{-2} \mathrm{Ga}$ dose at an ion energy of $100 \mathrm{keV}$. (a) As-implanted sample. (b) Sample annealed at $800^{\circ} \mathrm{C}$ RTA in the flowing $\mathrm{Ar}$ atmosphere.

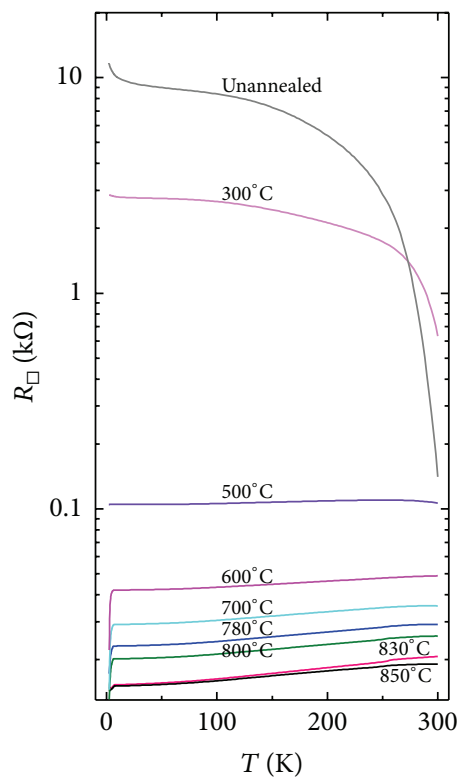

(a)

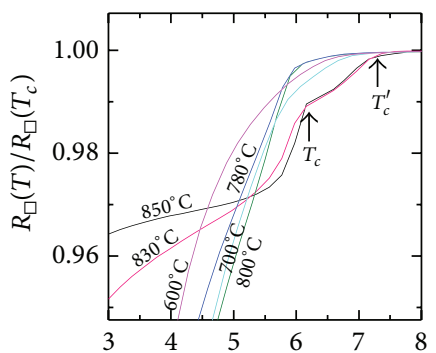

(b)

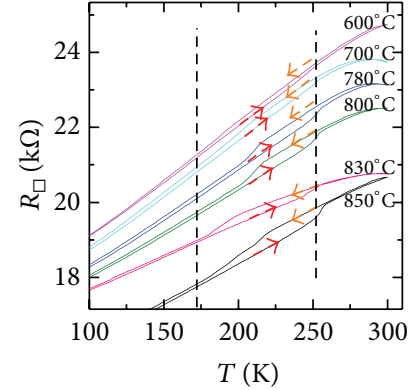

(c)

FIGURE 2: (a) $T$ dependence of the sheet resistance $R_{\square}$ for samples with the different annealing temperatures. (b) The enlarged plot of (a) near the superconducting transition temperature $T_{c}$. (c) The thermal hysteresis curves for the samples annealed at temperature window $\left(600^{\circ} \mathrm{C}-\right.$ $850^{\circ} \mathrm{C}$ ). The arrows show the direction of the thermal cycle.

on the surface of the sample because of the protection of the $\mathrm{SiO}_{2}$ capping as discussed before. But there are many discrete holes on the surface as a result of high dose implantation. For the as-implanted samples, the holes are discrete islands containing gallium; but for the samples annealed at $800^{\circ} \mathrm{C}$, the gallium islands inside the holes percolate which can be regarded as superconducting grains of gallium percolating in the normal state germanium matrix.

To clarify the properties of the different annealed samples, we performed the electronic transport measurements on those annealed samples. We contacted the electrodes by the bonding lines and carried out the measurements in the usual four-terminal geometry at the temperature from $2.5 \mathrm{~K}$ to $300 \mathrm{~K}$ in a physical property measurement system (PPMS). The resistance was measured by ETO option whose excitation current is $100 \mu \mathrm{A}$. The temperature dependence of resistance in the as-implanted state and annealed at various temperatures under Ar flowing atmosphere is presented in Figure 2.

Figure 2(a) is the $T$ dependence of the sheet resistance $R_{\square}$ for the unannealed and annealed samples. The $R_{\square}$ s decreases as the annealing temperature increases. The logarithmic scale of $R_{\square}$ here shows how dramatic the electronic transport can be influenced by the annealing temperature. For different annealing temperature, it shows a transition from the insulating to superconducting behavior. Upon cooling, the as-implanted samples show an increasing resistance, which could reflect intrinsic semiconductor property of Ge, while upon increasing the annealing temperature, the sheet resistance decreases, which could be due to the change of the discrete $\mathrm{Ga}$ islands to percolating ones as discussed above. 


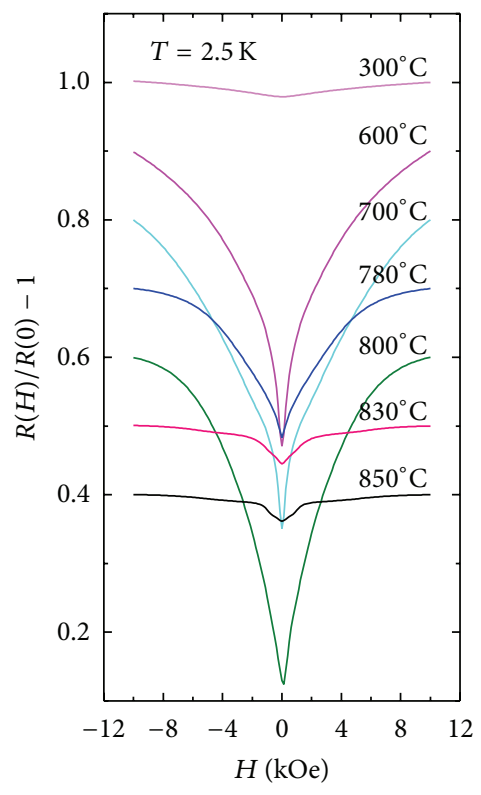

(a)

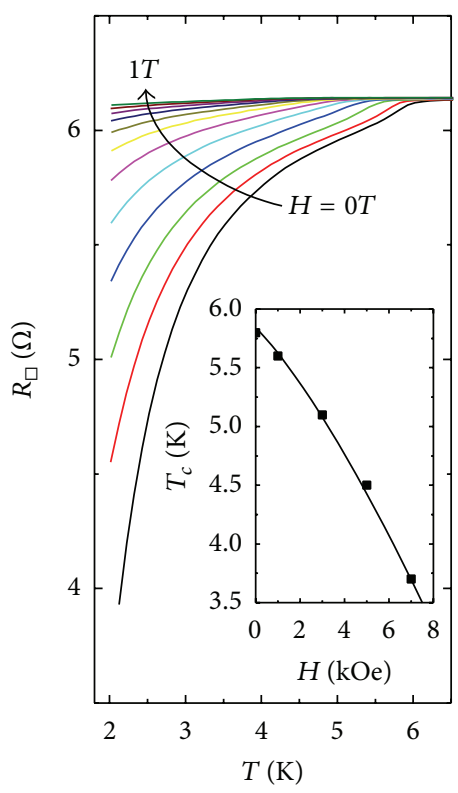

(b)

Figure 3: (a) The $H$ dependence of normalized magnetic resistance $(R(H) / R(0)-1)$ measured at $T=2.5 \mathrm{~K}$ for the samples annealed at temperature ranging from $300^{\circ} \mathrm{C}$ to $850^{\circ} \mathrm{C}$. (b) $T$ dependence of the sheet resistance $R_{\square}$ at various magnetic fields $H$ applied perpendicular to the surface for the samples annealed at $800^{\circ} \mathrm{C}$. The inset shows the field-temperature $(H-T)$ phase diagram.

At the annealing temperatures ranging from $600^{\circ} \mathrm{C}$ to $850^{\circ} \mathrm{C}$, the samples show a superconducting transition below $7 \mathrm{~K}$ as shown in Figure 2(b). There is a gradual drop of sheet resistance but zero resistance is not possible because of the discontinued superconducting path in the sample. When focusing on the low temperature transport carefully, we observed a detailed dependence of the superconducting properties on the annealing conditions. There is an optimal annealing temperature window $\left(600^{\circ} \mathrm{C}-800^{\circ} \mathrm{C}\right)$. Out of that temperature window, the superconductivity is weak. There are two critical temperatures in the samples with annealing temperature at $830^{\circ} \mathrm{C}$ and $850^{\circ} \mathrm{C}$.

It was shown in Figure 2(c) that the samples with superconducting behavior display a thermal hysteresis loop during the thermal cycle and the arrows mark the direction of those thermal cycles. The thermal hysteresis observed here shows the presence of the first order phase transition which could be attributed to the phase transition of the Ga grains from endothermic and exothermic process [9]. The gallium with exothermic process actually shows a similar superconducting transition temperature $[10,11]$ as the $T_{c}$ in Figure 2(b). The superconducting gallium grains actually play an important role in the process. Based on [9], the $\beta-\mathrm{Ga}, \gamma-\mathrm{Ga}$, and $\delta$ Ga show the similar hysteresis loop in the single-energy $\mathrm{X}$-ray absorption measurements and the reason is their endothermic and exothermic process during thermal cycle. So we attribute the hysteresis loop to the gallium and relate the superconductivity to the hysteresis loop. Particularly the $T_{c}$ of $\beta-\mathrm{Ga}$ is $6 \mathrm{~K}$. It therefore clarifies the reason that the superconducting behavior is related to thermal hysteresis as observed in this experiment.
To obtain the further understanding of the character of superconducting state, we measured the magnetic field $H$ dependence of the sheet resistance of the samples annealed from $300^{\circ} \mathrm{C}$ to $850^{\circ} \mathrm{C}$. The normalized magnetoresistance $(R(H) / R(0)-1)$ is plotted in Figure 3(a). (The data is shifted for clarity.) Here the $H$ is perpendicular to the $a b$ plane of samples. As shown in Figure 3(a), the samples show the largest magnetoresistance variation at the annealing temperature window between $600^{\circ} \mathrm{C}$ and $800^{\circ} \mathrm{C}$, which might suggest the strongest superconducting character there. We also performed the $T$ dependence of sheet resistance $R_{\square}$ measurement for the sample annealed at $800^{\circ} \mathrm{C}$ with the applied magnetic fields $H$ perpendicular to the $a b$-plane of the sample (Figure 3(b)). It is shown in Figure 3(b) that the superconducting transition is suppressed by the applied field $H$. We can define the critical temperature $T_{c}$ as the $2 \%$ drop of normal state resistance for the sample measured at different $H$. The inset in Figure 3(b) is the $H-T$ phase diagram of the samples. The $H_{c 2}(0 \mathrm{~K})$ at zero temperature was estimated as the $10 \%$ drop of $H_{c 2}$ at $2 \mathrm{~K}$, which is approximately $0.5 \mathrm{~T}$. Such a criterion is the same as the early work [6]. By using the standard theory, we can calculate the value of the GinzburgLandau coherence length $\xi_{\mathrm{GL}}$. We deduce the $\xi_{\mathrm{GL}}=26 \mathrm{~nm}$ via $\phi_{0}=2 \pi H_{c 2} \xi_{\mathrm{GL}}^{2}$, where $\phi_{0}$ is the magnetic flux quantum.

\section{Summary}

In summary, we have fabricated heavily Ga-doped Ge with a $30 \mathrm{~nm} \mathrm{SiO}$ cover layer. The ion implantation and subsequent RTA annealing were employed to form a Ga-doped layer at the $\mathrm{SiO}_{2} / \mathrm{Ge}$ interface. The RTA annealing enables $\mathrm{Ga}$ to 
redistribute in the sample and realize the superconducting circuits. Ga forms metallic precipitates in the Ge matrix. With increasing annealing temperature, the samples show a crossover from the insulator to the superconductor. The $T_{c}$ is the same with the gallium's transition temperature and the hysteresis loop is related to gallium. So, the electronic transport measurements indicate that the Ga-doped layer plays a leading role in the superconductivity.

\section{Conflict of Interests}

The authors declare that there is no conflict of interests regarding the publication of this paper.

\section{Acknowledgment}

This work is supported by the "Strategic Priority Research Program (B)" of the Chinese Academy of Sciences Grant no. XDB04040300.

\section{References}

[1] J. G. Bednorz and K. A. Müller, "Possible high $T_{c}$ superconductivity in the Ba-La-Cu-O system," Zeitschrift für Physik B: Condensed Matter, vol. 64, no. 2, pp. 189-193, 1986.

[2] Y. Kamihara, T. Watanabe, M. Hirano, and H. Hosono, "Ironbased layered superconductor $\mathrm{La}\left[\mathrm{O}_{1-x} \mathrm{~F}_{x}\right] \mathrm{FeAs}(\mathrm{x}=0.05-0.12)$ with $\mathrm{T}_{c}=26 \mathrm{~K}$," Journal of the American Chemical Society, vol. 130, no. 11, pp. 3296-3297, 2008.

[3] E. A. Ekimov, V. A. Sidorov, E. D. Bauer et al., "Superconductivity in diamond," Nature, vol. 428, no. 6982, pp. 542-545, 2004.

[4] E. Bustarret, C. Marcenat, P. Achatz et al., "Superconductivity in doped cubic silicon," Nature, vol. 444, no. 7118, pp. 465-468, 2006.

[5] T. Herrmannsdörfer, V. Heera, O. Ignatchik et al., "Superconducting state in a gallium-doped germanium layer at low temperatures," Physical Review Letters, vol. 102, no. 21, Article ID 217003, 2009.

[6] J. Fiedler, V. Heera, R. Skrotzki et al., "Superconducting Gaoverdoped Ge layers capped with $\mathrm{SiO}_{2}$ : structural and transport investigations," Physical Review B, vol. 85, no. 13, Article ID 134530, 2012.

[7] J. Fiedler, V. Heera, R. Skrotzki et al., "Superconducting films fabricated by high-fluence Ga implantation in Si," Physical Review B, vol. 83, no. 21, Article ID 214504, 2011.

[8] V. Heera, A. Mücklich, M. Posselt et al., "Heavily Ga-doped germanium layers produced by ion implantation and flash lamp annealing: structure and electrical activation," Journal of Applied Physics, vol. 107, no. 5, Article ID 053508, 2010.

[9] A. Di Cicco, "Phase transitions in confined gallium droplets," Physical Review Letters, vol. 81, no. 14, pp. 2942-2945, 1998.

[10] H. M. Jaeger, D. B. Haviland, A. M. Goldman, and B. G. Orr, "Threshold for superconductivity in ultrathin amorphous gallium films," Physical Review B, vol. 34, no. 7, pp. 4920-4923, 1986.

[11] H. Parr and J. Feder, "Superconductivity in beta-Phase Gallium," Physical Review B, vol. 7, no. 1, pp. 166-181, 1973. 

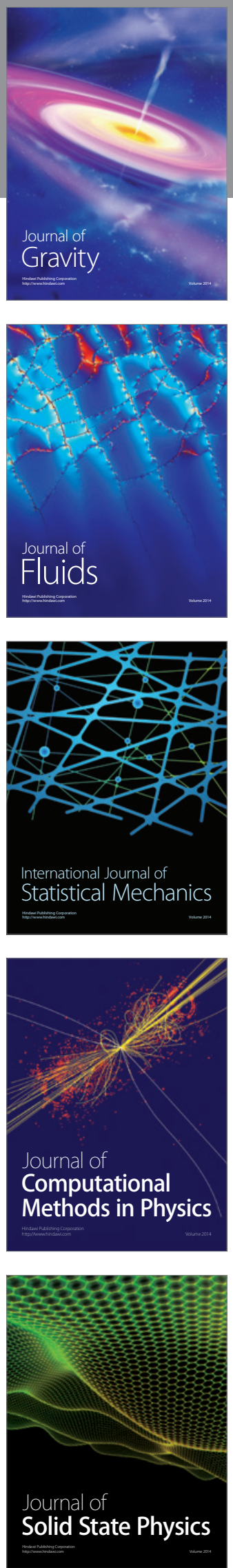

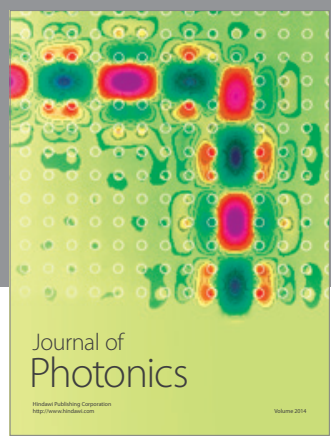

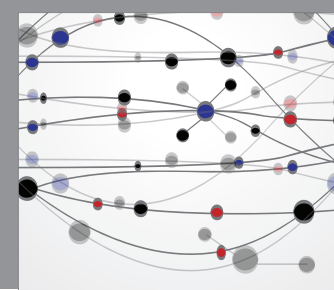

The Scientific World Journal

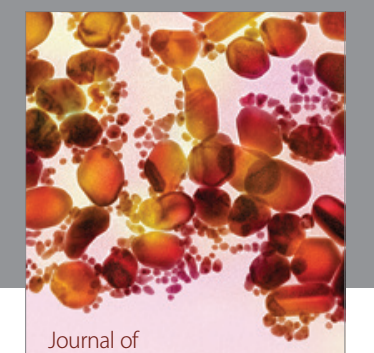

Soft Matter
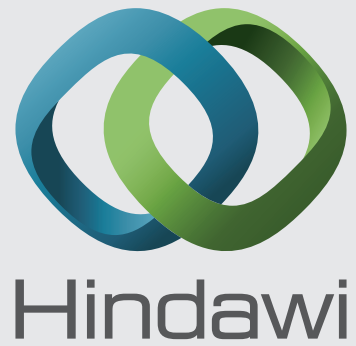

Submit your manuscripts at

http://www.hindawi.com
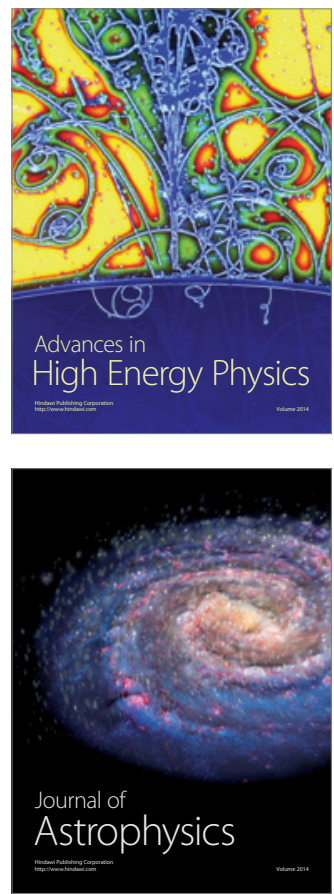
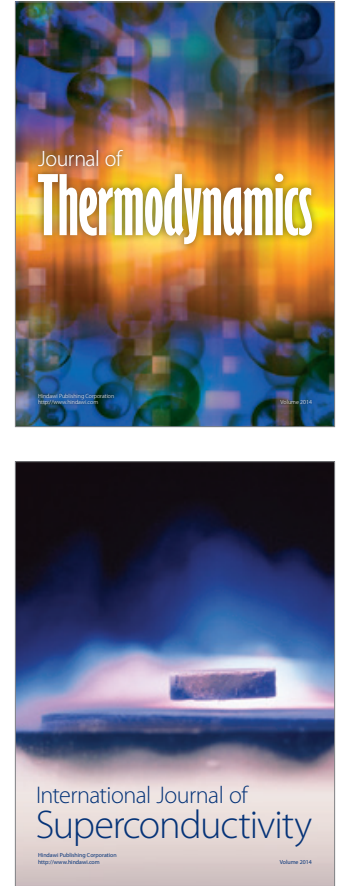
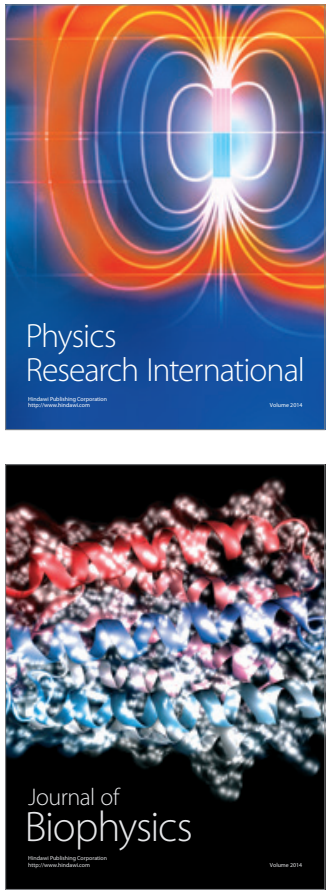
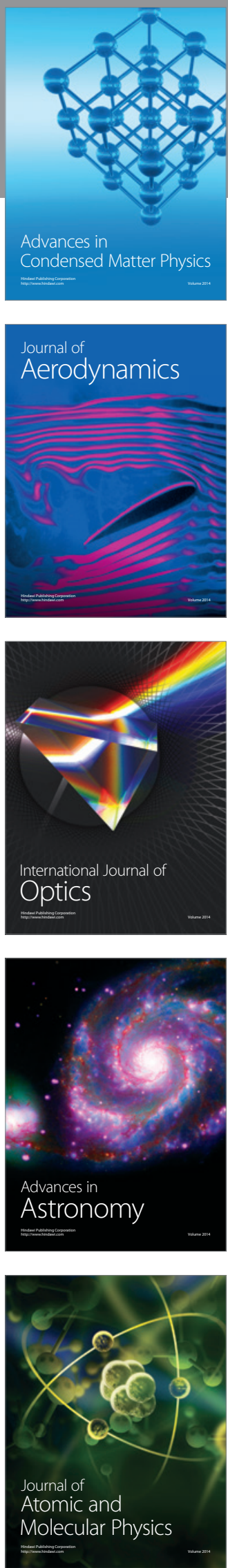